\title{
Atrial fibrilation in patients with systolic heart dysfunction and central vs. obstructive sleep apnea
}

\author{
Márcio Galindo Kiuchi*1 and Shaojie Chen ${ }^{2}$ \\ ${ }^{1}$ Division of Cardiac Surgery and Artificial Cardiac Stimulation, Department of Medicine, Hospital e Clínica São Gonçalo, São Gonçalo, RJ, Brazil \\ ${ }^{2}$ Department of Cardiology, Shanghai First People's Hospital, Shanghai Jiao Tong University School of Medicine, Shanghai, China
}

Central sleep apnea (CSA) is a subtype of Sleep-disordered breathing (SBD) with a repetitive pattern of cessations (or reductions) and resumptions (or increases) of respiratory effort not accompanied by significant upper-airway obstruction. Although CSA and obstructive sleep apnea (OSA) may share some of the same attributes, including similar underlying mechanisms resulting in unstable control of breathing, the clinical manifestations, sequelae, and treatment can differ significantly. Not infrequently, the two forms of SDB may be manifest in the same individual, further complicating management. Both central and obstructive events result in sleep disruption, hypoxemia, and increased overall sympathetic tone. Both OSA and CSA are associated with an increased prevalence of atrial fibrillation (AF) and ventricular arrhythmias. Congestive heart failure (CHF) complicated by CSA carries a greater risk of increased mortality [1] independent of other known risk factors such as impaired left ventricular ejection fraction (LVEF) [2].

Based on this information, we aim to compare the percentage of atrial fibrillation events recorded by the implanted dual chamber pacemakers in heart failure patients with CSA vs. OSA.

We selected 98 patients with heart failure, normal renal function, all of them having a pacemaker and CSA or OSA. The study was piloted in agreement with the Helsinki declaration and approved by the ethics committee of our institution. All patients signed the informed consent term before inclusion. This study was conducted at the Hospital e Clínica São Gonçalo, Rio de Janeiro, Brazil. Patients were recruited from January 2015 till January 2016 from the Arrhythmias and Artificial Cardiac Pacing Service of the same hospital. Patients with the combination of the following criteria were consecutively enrolled: (i) age between 18 and 70 years; (ii) implanted dual chamber pacemaker, without ischemia proved by myocardial scintigraphy or coronary angiography; (iii) to present CSA (defined as chronic respiratory disorder characterized by fluctuations in respiratory drive, resulting in the cessation of respiratory muscle activity and airflow during sleep [3]) or OSA (resulted when the lenient tissues around the upper airway collapse, obstructing air flow partly or completely, despite increased ventilator effort [4]) diagnosed by the polysomnography; (v) glomerular filtration rate estimated by the Chronic Kidney Disease Epidemiology Collaboration (CKD-EPI equation, eGFR [5] $>60 \mathrm{~mL} /$ $\mathrm{min} / 1.73 \mathrm{~m}^{2}$ without microalbuminuria); (vi) systolic heart dysfunction with left ventricular ejection fraction $<45 \%$; and (vii) the capacity to read, comprehend, and sign the informed consent form and attend the clinical tests. The patients that presented any of the subsequent criteria were excluded: (i) pregnancy; (ii) valvular disease with significant adverse sequelae; (iii) unstable angina, myocardial infarction, transient ischemic attack or stroke within the 6 months before the procedure; (iv) renovascular abnormalities; (v) psychiatric disease; (vi) the inability to be monitored clinically after the procedure; or (vii) a known addiction to drugs or alcohol that affects the intellect. The patients were separated into two groups: CSA $(n=42)$ and OSA $(n=56)$, both groups received continuous positive airway pressure (CPAP) treatment.

The results are expressed as a mean and standard deviation for normally distributed data and as median with interquartile range otherwise. All statistical tests were two-sided. Comparisons between two-paired values were performed with the paired t-test in cases of a Gaussian distribution and by the Wilcoxon test otherwise. Comparisons between more than two-paired values were made by repeatedmeasures analysis of variance or by Kruskal-Wallis analysis of variance as appropriate, complemented by a post-hoc test. Categorical variables were compared with Fisher's exact test. A P-value $<0.05$ was considered significant. Correlations between two variables were performed by Pearson's chi-square test in case of a Gaussian distribution and with the Spearman correlation test otherwise. All statistical analyses were performed using the program Graphpad Prism v 7.0 (Graphpad Software, La Jolla, CA, USA).

The general features and echocardiographic parameters at baseline of the 98 patients divided into two groups are listed in Table 1. The CSA group presented $73.8 \%$ of AF events and the OSA group showed $48.2 \%$ of AF records, with a Relative Risk $=1.944,95 \%$ Confidence Interval $=1.154$ to 3.467 , and $\mathrm{P}$ value $=0.0131$ (Figure 1$)$.

Our data show that in patients with systolic heart dysfunction with left ventricular ejection fraction $<45 \%$ and CSA the risk of AF events is higher than in patients with OSA, as the events recorded by the pacemakers.

\section{Conflict of interest}

None declared.

\section{Funding} Brazil.

This study was funded by Pacemed (US \$100,000), Rio de Janeiro,

Correspondence to: Márcio Galindo Kiuchi, MD, MSc, PhD, Division of Cardiac Surgery and Artificial Cardiac Stimulation, Department of Medicine, Hospital e Clínica São Gonçalo, Rua Cel. Moreira César, 138 - Centro, São Gonçalo, Rio de Janeiro 24440-400, Brazil, Tel/Fax: +55 (21) 26047744

Received: December 01, 2016; Accepted: December 21, 2016; Published: December 26, 2016 


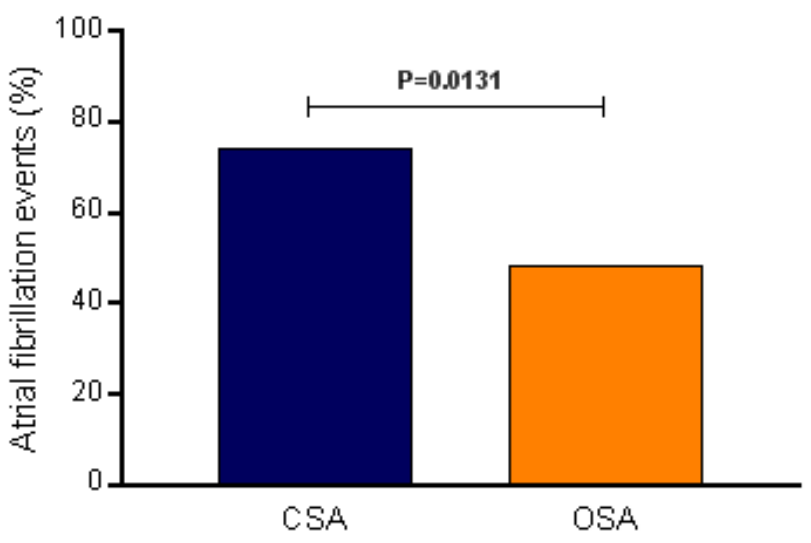

Figure 1. Atrial fibrillation events (\%) recorded by the pacemakers in the central sleep apnea (CSA, $n=42)$ and obstructive sleep apnea (OSA, $n=56)$.

\section{Acknowledgements}

The authors are grateful to all participants included in this study. The authors also thank Pacemed for stimulating the development of this study and for providing technical support.

\section{References}

1. Findley LJ, Zwillich CW, Ancoli-Israel S, Kripke D, Tisi G, et al. (1985) CheyneStokes breathing during sleep in patients with left ventricular heart failure. South Med J 78: 11-15. [Crossref]

2. Lanfranchi PA, Braghiroli A, Bosimini E, Mazzuero G, Colombo R, et al. (1999) Prognostic value of nocturnal Cheyne-Stokes respiration in chronic heart failure. Circulation 99: 1435-1440.

3. American Academy of Sleep Medicine (2005) International Classification of Sleep Disorders: Diagnostic and Coding Manual, 2nd ed. Westchester, IL.

4. Somers VK, White DP, Amin R, Abraham WT, Costa F, et al. (2008) Sleep apnea and cardiovascular disease: an American Heart Association/American College of Cardiology Foundation Scientific Statement from the American Heart Association Council for High Blood Pressure Research Professional Education Committee, Council on Clinical Cardiology, Stroke Council, and Council on Cardiovascular Nursing. J Am Coll Cardiol 52: 686-717.

5. Levey AS, Stevens LA, Schmid CH, Zhang YL, Castro AF III, et al. (2009) CKD-EPI (Chronic Kidney Disease Epidemiology Collaboration): A new equation to estimate glomerular filtration rate. Ann Intern Med: 604-612.

Copyright: ( 2016 Mirzaei R. This is an open-access article distributed under the terms of the Creative Commons Attribution License, which permits unrestricted use, distribution, and reproduction in any medium, provided the original author and source are credited. 